\title{
Pacific
}

Journal of

Mathematics

\section{A CASSON-LIN TYPE INVARIANT FOR LINKS}

ERIC HARPER AND Nikolai SAVElieV 


\title{
A CASSON-LIN TYPE INVARIANT FOR LINKS
}

\author{
ERIC HARPER AND NikOLAI SAVELIEV
}

\begin{abstract}
In 1992, Xiao-Song Lin constructed an invariant $h(K)$ of knots $K \subset S^{3}$ via a signed count of conjugacy classes of irreducible $S U(2)$ representations of $\pi_{1}\left(S^{3}-K\right)$ with trace-free meridians. Lin showed that $h(K)$ equals one half times the knot signature of $K$. Using methods similar to Lin's, we construct an invariant $h(L)$ of two-component links $L \subset S^{3}$. Our invariant is a signed count of conjugacy classes of projective $S U(2)$ representations of $\pi_{1}\left(S^{3}-L\right)$ with a fixed 2-cocycle and corresponding nontrivial $w_{2}$. We show that $h(L)$ is, up to a sign, the linking number of $L$.
\end{abstract}

\section{Introduction}

One of the characteristic features of the fundamental group of a closed 3-manifold is that its representation variety in a compact Lie group tends to be finite, in a properly understood sense. This has been a guiding principle for defining invariants of 3-manifolds ever since Casson defined his $\lambda$-invariant for integral homology 3 spheres via a signed count of the $S U(2)$ representations of the fundamental group, where signs were determined using Heegaard splittings.

Among numerous generalizations of Casson's construction, we will single out the invariant of knots in $S^{3}$ defined by Xiao-Song Lin [1992] via a signed count of SU(2) representations of the fundamental group of the knot exterior. The latter is a 3-manifold with nonempty boundary so the finiteness principle above only applies after one imposes a proper boundary condition. Lin's choice of boundary condition, namely, that all of the knot meridians are represented by trace-free $\mathrm{SU}(2)$ matrices, resulted in an invariant $h(K)$ of knots $K \subset S^{3}$. Lin further showed that $h(K)$ equals half the knot signature of $K$.

The signs in Lin's construction were determined using braid representations for knots. Austin (unpublished) and Heusener and Kroll [1998] extended Lin's construction by letting the meridians of the knot be represented by SU(2) matrices with

MSC2000: primary 57M25; secondary 57M05.

Keywords: link group, braid, projective representation.

Saveliev was partially supported by NSF grant 0305946 and the Max-Planck-Institut für Mathematik in Bonn, Germany. 
a fixed trace that need not be zero. Their construction gives a knot invariant that equals, for most choices of the trace, one half times the equivariant knot signature.

In this paper, we extend Lin's construction to two-component links $L$ in $S^{3}$. In essence, we replace the count of $\mathrm{SU}(2)$ representations with a count of projective $\mathrm{SU}(2)$ representations of $\pi_{1}\left(S^{3}-L\right)$, in the sense of [Ruberman and Saveliev 2004], with a fixed 2-cocycle representing a nontrivial element in the second group cohomology of $\pi_{1}\left(S^{3}-L\right)$. The resulting signed count is denoted by $h(L)$. The two main results of this paper are then as follows.

Theorem 1. For any two-component link $L \subset S^{3}$, the integer $h(L)$ is a well-defined invariant of $L$.

Theorem 2. For any two-component link $L=\ell_{1} \cup \ell_{2}$ in $S^{3}$, one has

$$
h(L)= \pm \operatorname{lk}\left(\ell_{1}, \ell_{2}\right)
$$

Our choice of the 2-cocycle imposes Lin's trace-free condition on us. This is in contrast to Lin's construction, where the choice of boundary condition seemed somewhat arbitrary. This also means one should not expect to extend our construction to $\mathrm{SU}(2)$ representations with nonzero trace boundary condition.

Shortly after Casson introduced his invariant for homology 3-spheres, Taubes [1990] gave a gauge theoretic description of it in terms of a signed count of flat SU(2) connections. After Lin's work, but before Heusener and Kroll, a gauge theoretic interpretation of the Lin invariant was given by Herald [1997]. He used this interpretation to define an extension of the Lin invariant, now known as the Herald-Lin invariant, to knots in arbitrary homology spheres, with arbitrary fixedtrace (possibly nonzero) boundary condition.

Another attractive feature of the gauge theoretic approach is that it can be used to produce ramified versions of the above invariants. Floer [1988] introduced the instanton homology theory whose Euler characteristic is twice the Casson invariant. We expect that our invariant will have a similar interpretation, perhaps along the lines of the knot instanton homology theory of Kronheimer and Mrowka [2008], which in turn is a variant of the orbifold Floer homology of Collin and Steer [1999]. We hope to discuss this elsewhere, together with possible extensions to links in homology spheres and to links of more than two components.

\section{Braids and representations}

Let $F_{n}$ be a free group of rank $n \geq 2$, with a fixed generating set $x_{1}, \ldots, x_{n}$. We will follow the conventions of [Long 1989] and define the $n$-string braid group $\mathscr{B}_{n}$ to be the subgroup of $\operatorname{Aut}\left(F_{n}\right)$ generated by the automorphisms $\sigma_{1}, \ldots, \sigma_{n-1}$, 
where the action of $\sigma_{i}$ is given by

$$
\begin{aligned}
\sigma_{i}: x_{i} & \mapsto x_{i+1}, \\
x_{i+1} & \mapsto\left(x_{i+1}\right)^{-1} x_{i} x_{i+1}, \\
x_{j} & \mapsto x_{j} \quad \text { for } j \neq i, i+1 .
\end{aligned}
$$

The natural homomorphism $\mathscr{B}_{n} \rightarrow S_{n}, \sigma \mapsto \bar{\sigma}$, onto the symmetric group on $n$ letters maps each generator $\sigma_{i}$ to the transposition $\bar{\sigma}_{i}=(i, i+1)$. A useful observation is that, for any $\sigma \in \operatorname{Aut}\left(F_{n}\right)$, one has

$$
\sigma\left(x_{i}\right)=w x_{\bar{\sigma}^{-1}(i)} w^{-1}
$$

for some word $w \in F_{n}$. Also $\sigma$ preserves the product $x_{1} \cdots x_{n}$, that is,

$$
\sigma\left(x_{1} \cdots x_{n}\right)=x_{1} \cdots x_{n} .
$$

2a. SU(2) representations. Consider the Lie group SU(2) of unitary two-by-two matrices with determinant one, that is, complex matrices

$$
\left(\begin{array}{rr}
u & v \\
-\bar{v} & \bar{u}
\end{array}\right)
$$

such that $u \bar{u}+v \bar{v}=1$. We will often identify $\mathrm{SU}(2)$ with the group $\mathrm{Sp}(1)$ of unit quaternions via

$$
\left(\begin{array}{rr}
u & v \\
-\bar{v} & \bar{u}
\end{array}\right) \mapsto u+v j \in \mathbb{H} .
$$

Let $R_{n}=\operatorname{Hom}\left(F_{n}, \mathrm{SU}(2)\right)$ be the space of SU(2) representations of $F_{n}$, and identify it with $\mathrm{SU}(2)^{n}$ by sending a representation $\alpha: F_{n} \rightarrow \mathrm{SU}(2)$ to the vector $\left(\alpha\left(x_{1}\right), \ldots, \alpha\left(x_{n}\right)\right)$ of $\mathrm{SU}(2)$ matrices. The above representation $\mathscr{P}_{n} \rightarrow \operatorname{Aut}\left(F_{n}\right)$ then gives rise to the representation

$$
\rho: \mathscr{B}_{n} \rightarrow \operatorname{Diff}\left(R_{n}\right)
$$

via $\rho(\sigma)(\alpha)=\alpha \circ \sigma^{-1}$. We will abbreviate $\rho(\sigma)$ to $\sigma$. We will also denote $X=$ $\left(X_{1}, \ldots, X_{n}\right) \in R_{n}$ and write $\sigma(X)=\left(\sigma(X)_{1}, \ldots, \sigma(X)_{n}\right)$.

Example. For any $\left(X_{1}, \ldots, X_{n}\right) \in R_{n}$, we have

$$
\sigma_{1}\left(X_{1}, X_{2}, X_{3}, \ldots, X_{n}\right)=\left(X_{1} X_{2} X_{1}^{-1}, X_{1}, X_{3}, \ldots, X_{n}\right) .
$$

2b. Extension to the wreath product $\mathbb{Z}_{2} 2_{B_{n}}$. The wreath product $\mathbb{Z}_{2} 2_{\mathscr{B}_{n}}$ is the semidirect product of $\mathscr{P}_{n}$ with $\left(\mathbb{Z}_{2}\right)^{n}$, where $\mathscr{B}_{n}$ acts on $\left(\mathbb{Z}_{2}\right)^{n}$ by permuting the coordinates, $\sigma\left(\varepsilon_{1}, \ldots, \varepsilon_{n}\right)=\left(\varepsilon_{\bar{\sigma}(1)}, \ldots, \varepsilon_{\bar{\sigma}(n)}\right)$. Thus the elements of $\mathbb{Z}_{2} \prec \mathscr{B}_{n}$ are the pairs $(\varepsilon, \sigma) \in\left(\mathbb{Z}_{2}\right)^{n} \times \mathscr{B}_{n}$, with the group multiplication law

$$
(\varepsilon, \sigma) \cdot\left(\varepsilon^{\prime}, \sigma^{\prime}\right)=\left(\varepsilon \sigma\left(\varepsilon^{\prime}\right), \sigma \sigma^{\prime}\right) .
$$


The representation (3) can be extended to a representation

$$
\rho: \mathbb{Z}_{2} \prec \mathscr{B}_{n} \rightarrow \operatorname{Diff}\left(R_{n}\right)
$$

by defining

$$
\rho(\varepsilon, \sigma)(X)=\varepsilon \cdot \sigma(X)=\left(\varepsilon_{1} \sigma(X)_{1}, \ldots, \varepsilon_{n} \sigma(X)_{n}\right),
$$

where the $\varepsilon_{i}$ are viewed as elements of the center $\mathbb{Z}_{2}=\{ \pm 1\}$ of $\mathrm{SU}(2)$. That (4) is a representation follows by a direct calculation after one observes that, by (1),

$$
\sigma(X)_{i}=A X_{\bar{\sigma}(i)} A^{-1} \text { for some } A \in \mathrm{SU}(2) .
$$

Again, we will abuse notation and write simply $\varepsilon \sigma$ for both $(\varepsilon, \sigma)$ and $\rho(\varepsilon, \sigma)$.

Example. For any $\left(X_{1}, \ldots, X_{n}\right) \in R_{n}$ and $\varepsilon=\left(\varepsilon_{1}, \ldots, \varepsilon_{n}\right) \in\left(\mathbb{Z}_{2}\right)^{n}$, we have

$$
\begin{array}{r}
\left(\varepsilon \sigma_{1}\right)\left(X_{1}, X_{2}, X_{3}, \ldots, X_{n}\right)=\left(\varepsilon_{1} X_{1} X_{2} X_{1}^{-1}, \varepsilon_{2} X_{1}, \varepsilon_{3} X_{3}, \ldots, \varepsilon_{n} X_{n}\right), \\
\sigma_{1}(\varepsilon X)=\sigma_{1}(\varepsilon) \sigma_{1}(X)=\left(\varepsilon_{2} X_{1} X_{2} X_{1}^{-1}, \varepsilon_{1} X_{1}, \varepsilon_{3} X_{3}, \ldots, \varepsilon_{n} X_{n}\right) .
\end{array}
$$

2c. Braids and link groups. The closure $\hat{\sigma}$ of a braid $\sigma \in \mathscr{B}_{n}$ is a link in $S^{3}$ with link group

$$
\left.\pi_{1}\left(S^{3}-\hat{\sigma}\right)=\left\langle x_{1}, \ldots, x_{n}\right| x_{i}=\sigma\left(x_{i}\right) \text { for } i=1, \ldots, n\right\rangle,
$$

where each $x_{i}$ represents a meridian of $\hat{\sigma}$. One can easily see that the fixed points of the diffeomorphism $\sigma: R_{n} \rightarrow R_{n}$ are representations $\pi_{1}\left(S^{3}-\hat{\sigma}\right) \rightarrow \mathrm{SU}(2)$. This paper grew out of the observation that a fixed point $\alpha=\left(\alpha\left(x_{1}\right), \ldots, \alpha\left(x_{n}\right)\right)$ of the map $\varepsilon \sigma: R_{n} \rightarrow R_{n}$ gives rise to a representation ad $\alpha: \pi_{1}\left(S^{3}-\hat{\sigma}\right) \rightarrow \mathrm{SO}(3)$ by composing with the adjoint representation ad : $\mathrm{SU}(2) \rightarrow \mathrm{SO}(3)$. Depending on $\varepsilon$, the representation ad $\alpha$ may or may not lift to an $\mathrm{SU}(2)$ representation, the obstruction being the second Stiefel-Whitney class $w_{2}(\operatorname{ad} \alpha) \in H^{2}\left(\pi_{1}\left(S^{3}-\hat{\sigma}\right) ; \mathbb{Z}_{2}\right)$.

\section{Definition of $h(\varepsilon \sigma)$}

Every link in $S^{3}$ is the closure $\hat{\sigma}$ of a braid $\sigma$; see [Alexander 1923]. Let $\sigma$ be a braid whose closure $\hat{\sigma}$ has two components. We will associate with it, for a carefully chosen $\varepsilon$, an integer $h(\varepsilon \sigma)$. We will prove in Section 4 that $h$ is an invariant of the link $\hat{\sigma}$.

3a. Choice of $\varepsilon$. The number of components of the link $\hat{\sigma}$ is exactly the number of cycles in the permutation $\bar{\sigma}$. We will be interested in two component links, that is, the closures of braids $\sigma$ with

$$
\bar{\sigma}=\left(i_{1} \ldots i_{m}\right)\left(i_{m+1} \ldots i_{n}\right) \text { for some } 1 \leq m \leq n-1 .
$$


Given such a braid $\sigma$, choose a vector $\varepsilon=\left(\varepsilon_{1}, \ldots, \varepsilon_{n}\right) \in\left(\mathbb{Z}_{2}\right)^{n}$ such that

$$
\varepsilon_{i_{1}} \cdots \varepsilon_{i_{m}}=\varepsilon_{i_{m+1}} \cdots \varepsilon_{i_{n}}=-1 .
$$

This choice of $\varepsilon$ is dictated by the following two considerations. First, we wish to preserve condition (2) in the form

$$
(\varepsilon \sigma)(X)_{1} \cdots(\varepsilon \sigma)(X)_{n}=X_{1} \cdots X_{n},
$$

and second, we want the fixed points $\alpha$ of the diffeomorphism $\varepsilon \sigma: R_{n} \rightarrow R_{n}$ to have nonzero $w_{2}(\operatorname{ad} \alpha)$.

Lemma 3.1. If $\alpha$ is a fixed point of $\varepsilon \sigma: R_{n} \rightarrow R_{n}$ with $\varepsilon$ as in (7), then $w_{2}(\operatorname{ad} \alpha) \neq 0$.

Proof. The class $w_{2}(\operatorname{ad} \alpha)$ is the obstruction to lifting ad $\alpha$ to an SU(2) representation. Extend $\alpha$ arbitrarily to a function $\alpha: \pi_{1}\left(S^{3}-\hat{\sigma}\right) \rightarrow \mathrm{SU}(2)$ lifting ad $\alpha$. Then $w_{2}(\operatorname{ad} \alpha)$ will vanish if and only if there is a function $\eta: \pi_{1}\left(S^{3}-\hat{\sigma}\right) \rightarrow \mathbb{Z}_{2}=\{ \pm 1\}$ such that $\eta \cdot \alpha$ is a representation. Suppose such a function exists, and write $\eta\left(x_{i}\right)=$ $\eta_{i}= \pm 1$. Also, assume without loss of generality that $\bar{\sigma}=(1 \ldots m)(m+1 \ldots n)$. It follows from (5) that to satisfy the relations $X_{i}=(\varepsilon \sigma)(X)_{i}$ we must have $\eta_{1}=\varepsilon_{1} \eta_{2}=\varepsilon_{1} \varepsilon_{2} \eta_{3}=\cdots=\varepsilon_{1} \cdots \varepsilon_{m} \eta_{1}=-\eta_{1}$, a contradiction with $\eta_{1}= \pm 1$.

This result for $w_{2}(\operatorname{ad} \alpha)$ can be sharpened using an algebraic topology lemma.

Lemma 3.2. Let $\hat{\sigma}$ be a link of two components. Then $H^{2}\left(\pi_{1}\left(S^{3}-\hat{\sigma}\right) ; \mathbb{Z}_{2}\right)$ is equal to $\mathbb{Z}_{2}$ if $\hat{\sigma}$ is nonsplit and is zero otherwise.

Proof. If $\hat{\sigma}$ is nonsplit, then $S^{3}-\hat{\sigma}$ is a $K(\pi, 1)$ by the sphere theorem; hence $H^{2}\left(\pi_{1}\left(S^{3}-\hat{\sigma}\right) ; \mathbb{Z}_{2}\right)=H^{2}\left(S^{3}-\hat{\sigma} ; \mathbb{Z}_{2}\right)=\mathbb{Z}_{2}$. If $\hat{\sigma}$ is split, then $K\left(\pi_{1}\left(S^{3}-\hat{\sigma}\right), 1\right)$ has the homotopy type of a one-point union of two circles and the result again follows.

Corollary 3.3. Let $\hat{\sigma}$ be a split link of two components, and let $\varepsilon$ be chosen as in (7). Then the diffeomorphism $\varepsilon \sigma: R_{n} \rightarrow R_{n}$ has no fixed points.

3b. The zero-trace condition. A naive way to define $h(\varepsilon \sigma)$ would be as the intersection number of the graph of $\varepsilon \sigma: R_{n} \rightarrow R_{n}$ with the diagonal in the product $R_{n} \times R_{n}$. One can observe though that, in addition to this intersection not being transversal, its points $(X, X)=\left(X_{1}, \ldots, X_{n}, X_{1}, \ldots, X_{n}\right)$ have the property that $\operatorname{tr} X_{1}=\cdots=\operatorname{tr} X_{n}=0$. This can be seen as follows.

Assume without loss of generality that $\bar{\sigma}=(1 \ldots m)(m+1 \ldots n)$. Then the relations $X=\varepsilon \sigma(X)$ together with (5) imply that

$$
\begin{aligned}
X_{1}= & \varepsilon_{1} \sigma(X)_{1}=\varepsilon_{1} A_{1} \cdot X_{\bar{\sigma}(1)} \cdot A_{1}^{-1}=\varepsilon_{1} A_{1} X_{2} A_{1}^{-1} \\
=\varepsilon_{1} A_{1} \cdot \varepsilon_{2} \sigma(X)_{2} \cdot A_{1}^{-1} & =\varepsilon_{1} \varepsilon_{2} A_{1} A_{2} \cdot X_{\bar{\sigma}(2)} \cdot A_{2}^{-1} A_{1}^{-1}=\cdots \\
& =\varepsilon_{1} \cdots \varepsilon_{m}\left(A_{1} \cdots A_{m}\right) \cdot X_{1} \cdot\left(A_{1} \ldots A_{m}\right)^{-1} .
\end{aligned}
$$


Since trace is conjugation invariant and $\varepsilon_{1} \cdots \varepsilon_{m}=-1$, we conclude that $\operatorname{tr} X_{1}=$ $\cdots=\operatorname{tr} X_{m}=0$. Similarly, $\operatorname{tr} X_{m+1}=\cdots=\operatorname{tr} X_{n}=0$.

Hence, in our definition we will restrict ourselves to the subset of $R_{n}$ consisting of $X=\left(X_{1}, \ldots, X_{n}\right)$ with $\operatorname{tr} X_{1}=\cdots=\operatorname{tr} X_{n}=0$. The nontransversality problem will be addressed below by factoring out the conjugation symmetry and lowering the dimension of the ambient manifold.

3c. The definition. The subset of $\mathrm{SU}(2)$ consisting of the matrices with zero trace is a conjugacy class in $\mathrm{SU}(2)$ diffeomorphic to $S^{2}$. Define

$$
Q_{n}=\left\{\left(X_{1}, \ldots, X_{n}\right) \in R_{n} \mid \operatorname{tr} X_{i}=0\right\} \subset R_{n},
$$

so that $Q_{n}$ is a manifold diffeomorphic to $\left(S^{2}\right)^{n}$. Also define

$$
H_{n}=\left\{\left(X_{1}, \ldots, X_{n}, Y_{1}, \ldots, Y_{n}\right) \in Q_{n} \times Q_{n} \mid X_{1} \cdots X_{n}=Y_{1} \cdots Y_{n}\right\} .
$$

This is no longer a manifold due to the presence of reducibles. We call a point $\left(X_{1}, \ldots, X_{n}, Y_{1}, \ldots, Y_{n}\right) \in Q_{n} \times Q_{n}$ reducible if all $X_{i}$ and $Y_{j}$ commute with each other or, equivalently, if there is a matrix $A \in \mathrm{SU}(2)$ such that the $A X_{i} A^{-1}$ and $A Y_{i} A^{-1}$ are diagonal matrices for $i=1, \ldots, n$. The subset $S_{n} \subset Q_{n} \times Q_{n}$ of reducibles is closed.

Lemma 3.4. $H_{n}^{*}=H_{n}-S_{n}$ is an open manifold of dimension $4 n-3$.

Proof. Consider the open manifold $\left(Q_{n} \times Q_{n}\right)^{*}=Q_{n} \times Q_{n}-S_{n}$ of dimension $4 n$ and the map $f:\left(Q_{n} \times Q_{n}\right)^{*} \rightarrow \mathrm{SU}(2)$ given by

$$
f\left(X_{1}, \ldots, X_{n}, Y_{1}, \ldots, Y_{n}\right)=X_{1} \cdots X_{n} Y_{n}^{-1} \cdots Y_{1}^{-1} .
$$

According to [Lin 1992, Lemma 1.5], this map has $1 \in \mathrm{SU}(2)$ as a regular value. Since $H_{n}^{*}=f^{-1}(1)$, the result follows.

Because of (5) and the fact that multiplication by $-1 \in \mathrm{SU}(2)$ preserves the zero trace condition, the representation (4) gives rise to a representation

$$
\rho: \mathbb{Z}_{2} 2 \mathscr{B}_{n} \rightarrow \operatorname{Diff}\left(Q_{n}\right) .
$$

Given $\varepsilon \sigma \in \mathbb{Z}_{2} 2 \mathscr{B}_{n}$ such that (6) and (7) are satisfied, consider two submanifolds of $Q_{n} \times Q_{n}$ : the graph $\Gamma_{\varepsilon \sigma}=\left\{(X, \varepsilon \sigma(X)) \mid X \in Q_{n}\right\}$ of $\varepsilon \sigma: Q_{n} \rightarrow Q_{n}$, and the diagonal $\Delta_{n}=\left\{(X, X) \mid X \in Q_{n}\right\}$. Note that both $\Gamma_{\varepsilon \sigma}$ and $\Delta_{n}$ are subsets of $H_{n}$; this is obvious for $\Delta_{n}$ and follows from (8) for $\Gamma_{\varepsilon \sigma}$.

Proposition 3.5. The intersection $\Gamma_{\varepsilon \sigma} \cap \Delta_{n} \subset H_{n}$ consists of irreducible representations.

Proof. Assume without loss of generality that $\bar{\sigma}=(1 \ldots m)(m+1 \ldots n)$, and suppose that $(X, X)=\left(X_{1}, \ldots, X_{n}, X_{1}, \ldots, X_{n}\right) \in \Gamma_{\varepsilon \sigma} \cap \Delta_{n}$ is reducible. Then all of the $X_{i}$ commute with each other, and in particular $\sigma(X)=\left(X_{\bar{\sigma}(1)}, \ldots, X_{\bar{\sigma}(n)}\right)$. 
The equality $X=\varepsilon \sigma(X)$ then implies that $X_{1}=\varepsilon_{1} X_{\bar{\sigma}(1)}=\varepsilon_{1} X_{2}=\varepsilon_{1} \varepsilon_{2} X_{\bar{\sigma}(2)}=$ $\cdots=\varepsilon_{1} \cdots \varepsilon_{m} X_{1}=-X_{1}$, which contradicts that $X_{1} \in \mathrm{SU}(2)$.

Let $\Gamma_{\varepsilon \sigma}^{*}=\Gamma_{\varepsilon \sigma} \cap H_{n}^{*}$ and $\Delta_{n}^{*}=\Delta_{n} \cap H_{n}^{*}$ be the irreducible parts of $\Gamma_{\varepsilon \sigma}$ and $\Delta_{n}$, respectively. They are both open submanifolds of $H_{n}^{*}$ of dimension $2 n$.

Corollary 3.6. The intersection $\Delta_{n}^{*} \cap \Gamma_{\varepsilon \sigma}^{*} \subset H_{n}^{*}$ is compact.

Proof. Proposition 3.5 implies that $\Delta_{n}^{*} \cap \Gamma_{\varepsilon \sigma}^{*}=\Delta_{n} \cap \Gamma_{\varepsilon \sigma}$, and the latter intersection is obviously compact since it is the intersection of two compact subsets of $H_{n}$.

The group $\mathrm{SO}(3)=\mathrm{SU}(2) /\{ \pm 1\}$ acts freely by conjugation on $H_{n}^{*}, \Delta_{n}^{*}$, and $\Gamma_{\varepsilon \sigma}^{*}$. Denote the resulting quotient manifolds by

$$
\hat{H}_{n}=H_{n}^{*} / \mathrm{SO}(3), \quad \hat{\Delta}_{n}=\Delta_{n}^{*} / \mathrm{SO}(3), \quad \text { and } \quad \hat{\Gamma}_{\varepsilon \sigma}=\Gamma_{\varepsilon \sigma}^{*} / \mathrm{SO}(3) .
$$

The dimension of $\hat{H}_{n}$ is $4 n-6$, and $\hat{\Delta}_{n}$ and $\hat{\Gamma}_{\varepsilon \sigma}$ are $(2 n-3)$-dimensional submanifolds. Since the intersection $\hat{\Delta}_{n} \cap \hat{\Gamma}_{\varepsilon \sigma}$ is compact, one can isotope $\hat{\Gamma}_{\varepsilon \sigma}$ into a submanifold $\widetilde{\Gamma}_{\varepsilon \sigma}$ using an isotopy with compact support so that $\hat{\Delta}_{n} \cap \widetilde{\Gamma}_{\varepsilon \sigma}$ consists of finitely many points. Define

$$
h(\varepsilon \sigma)=\#_{\hat{H}_{n}}\left(\hat{\Delta}_{n} \cap \widetilde{\Gamma}_{\varepsilon \sigma}\right)
$$

as the algebraic intersection number, where the orientations of $\hat{H}_{n}, \hat{\Delta}_{n}$, and $\widetilde{\Gamma}_{\varepsilon \sigma}$ are described in the next subsection. It is obvious that $h(\varepsilon \sigma)$ does not depend on the perturbation of $\hat{\Gamma}_{\varepsilon \sigma}$, so we will simply write

$$
h(\varepsilon \sigma)=\left\langle\hat{\Delta}_{n}, \hat{\Gamma}_{\varepsilon \sigma}\right\rangle_{\hat{H}_{n}} .
$$

3d. Orientations. Choose an arbitrary orientation of the copy of $S^{2} \subset \mathrm{SU}(2)$ cut out by the trace zero condition, and endow $Q_{n}=\left(S^{2}\right)^{n}$ and $Q_{n} \times Q_{n}$ with product orientations. The diagonal $\Delta_{n}$ and the graph $\Gamma_{\varepsilon \sigma}$ are naturally diffeomorphic to $Q_{n}$ via projection onto the first factor, and they are given the induced orientations. If we reverse the orientation of $S^{2}$, then the orientation of $Q_{n}$ is reversed if $n$ is odd. Hence the orientations of both $\Delta_{n}$ and $\Gamma_{\varepsilon \sigma}$ are reversed if $n$ is odd, while the orientation of $Q_{n} \times Q_{n}=\left(S^{2}\right)^{2 n}$ is preserved regardless of the parity of $n$.

Orient $\mathrm{SU}(2)$ by the standard basis $\{i, j, k\}$ in its Lie algebra $\mathfrak{s u}(2)$, and orient $H_{n}^{*}=f^{-1}(1)$ by applying the base-fiber rule to the map (9). The adjoint action of $\mathrm{SO}(3)$ on $S^{2} \subset \mathrm{SU}(2)$ is orientation preserving; hence the $\mathrm{SO}(3)$ quotients $\hat{H}_{n}, \hat{\Delta}_{n}$, and $\hat{\Gamma}_{\varepsilon \sigma}$ are orientable. We orient them using the base-fiber rule. The discussion in the previous paragraph shows that reversing orientation on $S^{2}$ may reverse the orientations of $\hat{\Delta}_{n}$ and $\hat{\Gamma}_{\varepsilon \sigma}$ but that it does not affect the intersection number $\left\langle\hat{\Delta}_{n}, \hat{\Gamma}_{\varepsilon \sigma}\right\rangle_{\hat{H}_{n}}$. 


\section{The link invariant $h$}

In this section, we will prove Theorem 1 . This will be accomplished by proving that $h(\varepsilon \sigma)$ is independent first of $\varepsilon$ and then of $\sigma$.

4a. Independence of $\boldsymbol{\varepsilon}$. We will first show that, for a fixed $\sigma$ whose closure $\hat{\sigma}$ is a link of two components, $h(\varepsilon \sigma)$ is independent of the choice of $\varepsilon$ as long as $\varepsilon$ satisfies (7).

Proposition 4.1. Let $\varepsilon$ and $\varepsilon^{\prime}$ be such that (7) is satisfied. Then $h(\varepsilon \sigma)=h\left(\varepsilon^{\prime} \sigma\right)$.

Proof. Assume without loss of generality that $\bar{\sigma}=(1 \ldots m)(m+1 \ldots n)$ and let $\varepsilon=\left(\varepsilon_{1}, \ldots, \varepsilon_{n}\right)$ and $\varepsilon^{\prime}=\left(\varepsilon_{1}^{\prime}, \ldots, \varepsilon_{n}^{\prime}\right)$. Define $\delta=\left(\delta_{1}, \ldots, \delta_{n}\right)$ as the vector in $\left(\mathbb{Z}_{2}\right)^{n}$ with coordinates

$$
\delta_{1}=1 \quad \text { and } \quad \delta_{k+1}=\delta_{k} \varepsilon_{k} \varepsilon_{k}^{\prime} \quad \text { for } k=1, \ldots, n-1,
$$

and define the involution $\tau: Q_{n} \rightarrow Q_{n}$ by the formula

$$
\tau(X)=\delta X=\left(\delta_{1} X_{1}, \delta_{2} X_{2}, \ldots, \delta_{n} X_{n}\right) .
$$

Recall that $Q_{n}=\left(S^{2}\right)^{n}$ so that $\tau$ is a diffeomorphism that restricts to each of the factors $S^{2}$ as either the identity or the antipodal map. In particular, $\tau$ need not be orientation preserving.

The map $\tau \times \tau: Q_{n} \times Q_{n} \rightarrow Q_{n} \times Q_{n}$ obviously preserves the irreducibility condition and commutes with the $\mathrm{SO}(3)$ action. It gives rise to an orientation preserving automorphism of $\hat{H}_{n}$, which will again be called $\tau \times \tau$. It is clear that $(\tau \times \tau)\left(\hat{\Delta}_{n}\right)=\hat{\Delta}_{n}$. It is also true that $(\tau \times \tau)\left(\hat{\Gamma}_{\varepsilon \sigma}\right)=\hat{\Gamma}_{\varepsilon^{\prime} \sigma}$, which can be seen as follows. Write a pair $(\delta X, \delta \varepsilon \sigma(X))$ whose conjugacy class belongs to $(\tau \times \tau)\left(\hat{\Gamma}_{\varepsilon \sigma}\right)$ as

$$
(\delta X, \delta \varepsilon \sigma(X))=(\delta X, \delta \varepsilon \sigma(\delta \delta X))=(\delta X, \delta \varepsilon \sigma(\delta) \sigma(\delta X))
$$

using the multiplication law in the group $\mathbb{Z}_{2} \mathscr{B}_{n}$. The conjugacy class of this pair belongs to $\Gamma_{\varepsilon^{\prime} \sigma}$ if and only if $\delta \varepsilon \sigma(\delta)=\varepsilon^{\prime}$. That this condition holds can be verified directly from the definition of $\delta$.

Recall that the orientations of $\hat{\Delta}_{n}, \hat{\Gamma}_{\varepsilon \sigma}$, and $\hat{\Gamma}_{\varepsilon^{\prime} \sigma}$ are induced by the orientation of $Q_{n}$. Therefore, the maps $\tau \times \tau: \hat{\Delta}_{n} \rightarrow \hat{\Delta}_{n}$ and $\tau \times \tau: \hat{\Gamma}_{\varepsilon \sigma} \rightarrow \hat{\Gamma}_{\varepsilon^{\prime} \sigma}$ are either both orientation preserving or both orientation reversing depending on whether $\tau: Q_{n} \rightarrow Q_{n}$ preserves or reverses orientation. Hence we have

$$
\begin{aligned}
h(\varepsilon \sigma)=\left\langle\hat{\Delta}_{n}, \hat{\Gamma}_{\varepsilon \sigma}\right\rangle_{\hat{H}_{n}} & =\left\langle(\tau \times \tau)\left(\hat{\Delta}_{n}\right),(\tau \times \tau)\left(\hat{\Gamma}_{\varepsilon \sigma}\right)\right\rangle_{(\tau \times \tau)\left(\hat{H}_{n}\right)} \\
& =\left\langle\hat{\Delta}_{n}, \hat{\Gamma}_{\varepsilon^{\prime} \sigma}\right\rangle_{\hat{H}_{n}}=h\left(\varepsilon^{\prime} \sigma\right) .
\end{aligned}
$$

From now on, we will drop $\varepsilon$ from the notation and simply write $h(\sigma)$ for $h(\varepsilon \sigma)$ assuming that a choice of $\varepsilon$ satisfying (7) has been made. 
4b. Independence of $\sigma$. In this section, we will show that $h(\sigma)$ only depends on the link $\hat{\sigma}$, not on a particular choice of braid $\sigma$, by verifying that $h$ is preserved under Markov moves. We will follow the proof of [Lin 1992, Theorem 1.8], which goes through with little change once the right $\varepsilon$ are chosen.

Recall that two braids $\alpha \in \mathscr{B}_{n}$ and $\beta \in \mathscr{B}_{m}$ have isotopic closures $\hat{\alpha}$ and $\hat{\beta}$ if and only if one braid can be obtained from the other by a finite sequence of Markov moves; see for instance [Birman 1974]. A type 1 Markov move replaces $\sigma \in \mathscr{B}_{n}$ by $\xi^{-1} \sigma \xi \in \mathscr{B}_{n}$ for any $\xi \in \mathscr{B}_{n}$. A type 2 Markov move means replacing $\sigma \in \mathscr{B}_{n}$ by $\sigma_{n}^{ \pm 1} \sigma \in \mathscr{B}_{n+1}$, or the inverse of this operation.

Proposition 4.2. The invariant $h(\sigma)$ is preserved by type 1 Markov moves.

Proof. Let $\xi, \sigma \in \mathscr{B}_{n}$ and assume as usual that $\bar{\sigma}=(1 \ldots m)(m+1 \ldots n)$. Then

$$
\overline{\xi^{-1} \sigma \xi}=(\bar{\xi}(1) \ldots \bar{\xi}(m))(\bar{\xi}(m+1) \ldots \bar{\xi}(n))
$$

has the same cycle structure as $\bar{\sigma}$. To compute $h\left(\xi^{-1} \sigma \xi\right)$, we will make a choice of $\varepsilon \in\left(\mathbb{Z}_{2}\right)^{n}$ that satisfies condition (7) with respect to the braid $\xi^{-1} \sigma \xi$, that is, $\varepsilon_{\bar{\xi}(1)} \cdots \varepsilon_{\bar{\xi}(m)}=\varepsilon_{\bar{\xi}(m+1)} \cdots \varepsilon_{\bar{\xi}(n)}=-1$.

The braid $\xi$ gives rise to the map $\xi: Q_{n} \rightarrow Q_{n}$. It acts by permutation and conjugation on the $S^{2}$ factors in $Q_{n}$; hence it is orientation preserving (we use the fact that $S^{2}$ is even-dimensional). It induces an orientation-preserving map $\xi \times \xi: Q_{n} \times Q_{n} \rightarrow Q_{n} \times Q_{n}$, which preserves the irreducibility condition and commutes with the $\mathrm{SO}(3)$ action. Equation (2) then ensures that we have a welldefined orientation-preserving automorphism $\xi \times \xi: \hat{H}_{n} \rightarrow \hat{H}_{n}$.

That this automorphism preserves the diagonal, $(\xi \times \xi)\left(\hat{\Delta}_{n}\right)=\hat{\Delta}_{n}$, is obvious. Concerning the graphs, let $\left(X, \varepsilon \xi^{-1} \sigma \xi(X)\right) \in \hat{\Gamma}_{\varepsilon \xi^{-1} \sigma \xi}$; then

$(\xi \times \xi)\left(X, \varepsilon \xi^{-1} \sigma \xi(X)\right)=\left(\xi(X), \xi\left(\varepsilon \xi^{-1} \sigma \xi(X)\right)\right)=(\xi(X), \xi(\varepsilon) \sigma(\xi(X))) \in \hat{\Gamma}_{\xi(\varepsilon) \sigma}$.

Therefore, $(\xi \times \xi)\left(\hat{\Gamma}_{\varepsilon \xi^{-1} \sigma \xi}\right)=\hat{\Gamma}_{\xi(\varepsilon) \sigma}$. Since $\xi: Q_{n} \rightarrow Q_{n}$ is orientation preserving, the identifications above of the diagonals and graphs via $\xi \times \xi$ are also orientation preserving.

Observe that $\xi(\varepsilon)_{i}=\varepsilon_{\bar{\xi}(i)}$. Hence $\xi(\varepsilon)$ satisfies (7) with respect to $\sigma$ and thus can be used to compute $h(\sigma)$. The argument is completed by the calculation

$$
\begin{aligned}
h\left(\xi^{-1} \sigma \xi\right)=\left\langle\hat{\Delta}_{n}, \hat{\Gamma}_{\varepsilon \xi^{-1} \sigma \xi}\right\rangle_{\hat{H}_{n}} & =\left\langle(\xi \times \xi)\left(\hat{\Delta}_{n}\right),(\xi \times \xi)\left(\hat{\Gamma}_{\varepsilon \xi^{-1} \sigma \xi}\right)\right\rangle_{(\xi \times \xi)\left(\hat{H}_{n}\right)} \\
& =\left\langle\hat{\Delta}_{n}, \hat{\Gamma}_{\xi(\varepsilon) \sigma}\right\rangle_{\hat{H}_{n}}=h(\sigma) .
\end{aligned}
$$

Proposition 4.3. The invariant $h(\sigma)$ is preserved by type 2 Markov moves. 
Proof. Given $\sigma \in \mathscr{B}_{n}$ and $\varepsilon$ satisfying (7), change $\sigma$ to $\sigma_{n} \sigma \in \mathscr{B}_{n+1}$ and let $\varepsilon^{\prime}=\sigma_{n}(\varepsilon, 1)$. If $X=\left(X_{1}, \ldots, X_{n}\right)$ and $\varepsilon=\left(\varepsilon_{1}, \ldots, \varepsilon_{n}\right)$, then

$$
\begin{aligned}
\left(\sigma_{n} \sigma\right)\left(X, X_{n+1}\right) & =\sigma_{n}\left(\sigma(X), X_{n+1}\right) \\
& =\left(\sigma(X)_{1}, \ldots, \sigma(X)_{n-1}, \sigma(X)_{n} X_{n+1} \sigma(X)_{n}^{-1}, \sigma(X)_{n}\right)
\end{aligned}
$$

and $\varepsilon^{\prime}=\left(\varepsilon_{1}, \ldots, \varepsilon_{n-1}, 1, \varepsilon_{n}\right)$. In particular, $\varepsilon^{\prime}$ satisfies (7) with respect to $\sigma_{n} \sigma$. Consider the embedding $g: Q_{n} \times Q_{n} \rightarrow Q_{n+1} \times Q_{n+1}$ given by

$$
g\left(X_{1}, \ldots, X_{n}, Y_{1}, \ldots, Y_{n}\right)=\left(X_{1}, \ldots, X_{n}, Y_{n}, Y_{1}, \ldots, Y_{n}, Y_{n}\right) .
$$

One can easily see that $g\left(H_{n}\right) \subset H_{n+1}$ and that $g$ commutes with the conjugation, thus giving rise to an embedding $\hat{g}: \hat{H}_{n} \rightarrow \hat{H}_{n+1}$. A straightforward calculation using the formulas above for $\sigma_{n} \sigma$ and $\varepsilon^{\prime}$ then shows that

$$
\hat{g}\left(\hat{\Delta}_{n}\right) \subset \hat{\Delta}_{n+1}, \quad \hat{g}\left(\hat{\Gamma}_{\varepsilon \sigma}\right) \subset \hat{\Gamma}_{\varepsilon^{\prime} \sigma_{n} \sigma}, \quad \text { and } \quad \hat{g}\left(\hat{\Delta}_{n} \cap \hat{\Gamma}_{\varepsilon \sigma}\right)=\hat{\Delta}_{n+1} \cap \hat{\Gamma}_{\varepsilon^{\prime} \sigma_{n} \sigma} .
$$

Now one can achieve all the necessary transversalities and match the orientations in exactly the same way as in the second half of the proof of [Lin 1992, Theorem 1.8]. This shows that $h\left(\sigma_{n} \sigma\right)=h(\sigma)$. The proof that $h\left(\sigma_{n}^{-1} \sigma\right)=h(\sigma)$ is similar.

\section{The invariant $h(\sigma)$ as the linking number}

In this section we will prove Theorem 2 , that is, show that for any link $\hat{\sigma}=\ell_{1} \cup \ell_{2}$ of two components, one has $h(\sigma)= \pm \operatorname{lk}\left(\ell_{1}, \ell_{2}\right)$. Our strategy will be to show that the invariant $h(\sigma)$ and the linking number $\operatorname{lk}\left(\ell_{1}, \ell_{2}\right)$ change according to the same rule as we change a crossing between two strands from two different components of $\hat{\sigma}=\ell_{1} \cup \ell_{2}$ (the link $\hat{\sigma}$ will need to be oriented for that, although a particular choice of orientation will not matter). After changing finitely many such crossings, we will arrive at a split link, for which both the invariant $h(\sigma)$ and the linking number $\operatorname{lk}\left(\ell_{1}, \ell_{2}\right)$ vanish; see Corollary 3.3. The change of crossing as above obviously changes the linking number by \pm 1 . To calculate the effect of the crossing change on $h(\sigma)$, we will follow [Lin 1992] and reduce the problem to a calculation in the pillowcase $\hat{H}_{2}$.

5a. The pillowcase. We begin with a geometric description of $\hat{H}_{2}$ as a pillowcase; compare with [Lin 1992, Lemma 1.2]. Remember that

$$
H_{2}=\left\{\left(X_{1}, X_{2}, Y_{1}, Y_{2}\right) \in Q_{2} \times Q_{2} \mid X_{1} X_{2}=Y_{1} Y_{2}\right\} .
$$

We will use the identification of $\mathrm{SU}(2)$ with $\mathrm{Sp}(1)$ when convenient. Since $X_{2}$ is trace free, we may assume that $X_{2}=i$ after conjugation. Conjugating by $e^{i \varphi}$ will 
not change $X_{2}$ but, for an appropriate choice of $\varphi$, will make $X_{1}$ into

$$
X_{1}=\left(\begin{array}{cc}
i r & u \\
-u & -i r
\end{array}\right)
$$

where both $r$ and $u$ are real, and $u$ is also nonnegative. Since $r^{2}+u^{2}=1$, we can write $r=\cos \theta$ and $u=\sin \theta$ for a unique $\theta$ such that $0 \leq \theta \leq \pi$. In quaternionic language, $X_{1}=i e^{-k \theta}$ with $0 \leq \theta \leq \pi$. Similarly, the condition $\operatorname{tr}\left(Y_{2}\right)=\operatorname{tr}\left(Y_{1}^{-1} X_{1} X_{2}\right)=0$ implies that $Y_{1}=i e^{-k \psi}$, this time with $-\pi \leq \psi \leq \pi$. To summarize,

$$
X_{1}=i e^{-k \theta}, \quad X_{2}=i, \quad Y_{1}=i e^{-k \psi}, \quad Y_{2}=i e^{-k(\psi-\theta)} .
$$

Thus $\hat{H}_{2}$ is parametrized by the rectangle $[0, \pi] \times[-\pi, \pi]$, with proper identifications along the edges and with the reducibles removed. The reducibles occur when both $\theta$ and $\psi$ are multiples of $\pi$, and hence $\hat{H}_{2}$ is a 2-sphere with the points $A=(0,0), B=(\pi, 0), A^{\prime}=(0, \pi)$, and $B^{\prime}=(\pi, \pi)$ removed; see Figure 1 . According to [Lin 1992], the orientation on the front sheet of $\hat{H}_{2}$ coincides with the standard orientation on the $(\theta, \psi)$ plane.

Example. Let $\sigma=\sigma_{1}^{2}$, so that $\hat{\sigma}=\ell_{1} \cup \ell_{2}$ is the Hopf link with $\operatorname{lk}\left(\ell_{1}, \ell_{2}\right)= \pm 1$. To calculate $h(\sigma)$, we let $\varepsilon=(-1,-1)$, the only available choice satisfying (7), and consider the submanifolds $\hat{\Delta}_{2}$ and $\hat{\Gamma}_{\varepsilon \sigma}$ of $\hat{H}_{2}$. We have, in quaternionic notation, $\hat{\Delta}_{2}=\left\{\left(i e^{-k \theta}, i, i e^{-k \theta}, i\right)\right\}$, which is the diagonal $\psi=\theta$ in the pillowcase. A straightforward calculation shows that $\hat{\Gamma}_{\varepsilon \sigma} \subset \hat{H}_{2}$ is given by $\psi=3 \theta-\pi$. As can be seen in Figure 1, the intersection $\hat{\Delta}_{2} \cap \hat{\Gamma}_{\varepsilon \sigma}$ consists of one point coming with a sign. Hence $h\left(\sigma_{1}^{2}\right)= \pm 1$, which is consistent with the fact that $\operatorname{lk}\left(\ell_{1}, \ell_{2}\right)= \pm 1$.

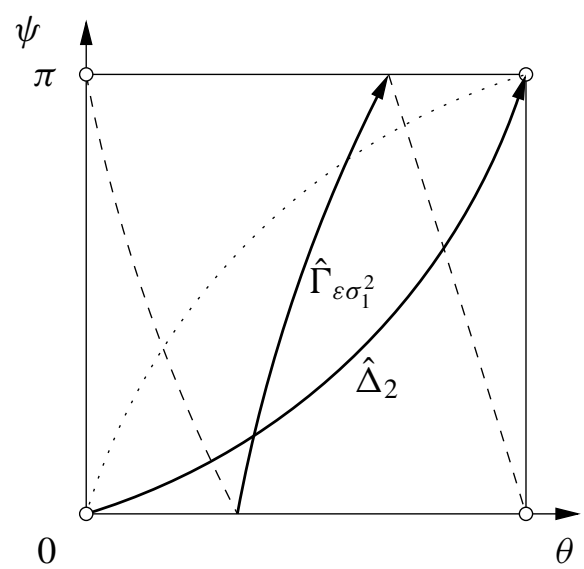

Figure 1. The pillowcase. 
Example. Let $\sigma=\sigma_{1}^{2 n}$. Then arguing as above one can show that $\hat{\Gamma}_{\varepsilon \sigma} \subset \hat{H}_{2}$ is given by $\psi=(2 n+1) \theta-\pi$. In this case there are $n$ intersection points all of which come with the same sign. This shows that $h\left(\sigma_{1}^{2 n}\right)= \pm n$, which is again consistent with the fact that $\operatorname{lk}\left(\ell_{1}, \ell_{2}\right)= \pm n$.

5b. The difference cycle. Fix an orientation on a given two component link $\hat{\sigma}$. A particular choice of orientation will not matter because we are only interested in identifying $h(\sigma)$ with the linking number $\operatorname{lk}\left(\ell_{1}, \ell_{2}\right)$ up to sign. We wish to change one of the crossings between the two components of $\hat{\sigma}$. Using a sequence of first Markov moves, we may assume that the first two strands of $\sigma$ belong to two different components of $\hat{\sigma}$, and that the crossing change occurs between these two strands. Furthermore, we may assume that the crossing change makes $\sigma$ into $\sigma_{1}^{ \pm 2} \sigma$, where the sign depends on the type of the crossing we change. Note that the braid $\sigma_{1}^{ \pm 2} \sigma$ has the same permutation type as $\sigma$; in particular, its closure is a link of two components. In fact, if we let $\sigma^{\prime}=\sigma_{1}^{-2} \sigma$, then

$$
h\left(\sigma_{1}^{-2} \sigma\right)-h(\sigma)=h\left(\sigma^{\prime}\right)-h\left(\sigma_{1}^{2} \sigma^{\prime}\right)=-\left(h\left(\sigma_{1}^{2} \sigma^{\prime}\right)-h\left(\sigma^{\prime}\right)\right) ;
$$

hence we only need to understand the difference $h\left(\sigma_{1}^{2} \sigma\right)-h(\sigma)$. Let us fix $\varepsilon=$ $(-1,-1,1, \ldots, 1)$. Since $\sigma_{1}^{2}$ and $\varepsilon$ commute, we have

$$
\begin{aligned}
h\left(\sigma_{1}^{2} \sigma\right)-h(\sigma) & =\left\langle\hat{\Delta}_{n}, \hat{\Gamma}_{\varepsilon \sigma_{1}^{2} \sigma}\right\rangle-\left\langle\hat{\Delta}_{n}, \hat{\Gamma}_{\varepsilon \sigma}\right\rangle \\
& =\left\langle\hat{\Gamma}_{\sigma_{1}^{-2}}, \hat{\Gamma}_{\varepsilon \sigma}\right\rangle-\left\langle\hat{\Delta}_{n}, \hat{\Gamma}_{\varepsilon \sigma}\right\rangle=\left\langle\hat{\Gamma}_{\sigma_{1}^{-2}}-\hat{\Delta}_{n}, \hat{\Gamma}_{\varepsilon \sigma}\right\rangle,
\end{aligned}
$$

where all intersection numbers are taken in $\hat{H}_{n}$. This leads us to consider the difference cycle $\hat{\Gamma}_{\sigma_{1}^{-2}}-\hat{\Delta}_{n}$ that is carried by $\hat{H}_{n}$. The next step in our argument will be to reduce the analysis of the intersection above to an intersection theory in the pillowcase $\hat{H}_{2}$.

5c. The pillowcase reduction. We consider the subset $V_{n} \subset H_{n}$ consisting of all $\left(X_{1}, \ldots, X_{n}, Y_{1}, \ldots, Y_{n}\right) \in H_{n}$ such that $X_{k}=Y_{k}$ for $k=3, \ldots, n$. Equivalently, $V_{n}$ consists of all $\left(X_{1}, \ldots, X_{n}, Y_{1}, \ldots, Y_{n}\right) \in Q_{n} \times Q_{n}$ such that $\left(X_{1}, X_{2}, Y_{1}, Y_{2}\right) \in H_{2}$ and $X_{k}=Y_{k}$ for all $k=3, \ldots, n$. Therefore, $V_{n}$ can be identified as

$$
V_{n}=H_{2} \times \Delta_{n-2} \subset\left(Q_{2} \times Q_{2}\right) \times\left(Q_{n-2} \times Q_{n-2}\right) .
$$

Lemma 5.1. The quotient $\hat{V}_{n}=\left(H_{2}^{*} \times \Delta_{n-2}\right) / \mathrm{SO}(3)$ is a submanifold of $\hat{H}_{n}$ of dimension $2 n-2$.

Proof. Since $H_{2}^{*}$ and $\Delta_{n-2}$ are smooth manifolds of dimensions 5 and $2 n-4$, respectively, and their product contains no reducibles, the statement follows.

Lemma 5.2. The difference cycle $\hat{\Gamma}_{\sigma_{1}^{-2}}-\hat{\Delta}_{n}$ is carried by $\hat{V}_{n}$. 
Proof. Observe that neither $\hat{\Gamma}_{\sigma_{1}^{-2}}$ nor $\hat{\Delta}_{n}$ are subsets of $\hat{V}_{n}$. However, their portions that do not fit in $\hat{V}_{n}$,

$$
\hat{\Gamma}_{\sigma_{1}^{-2}}-\left(\hat{\Gamma}_{\sigma_{1}^{-2}} \cap \hat{V}_{n}\right) \quad \text { and } \quad \hat{\Delta}_{n}-\left(\hat{\Delta}_{n} \cap \hat{V}_{n}\right),
$$

are exactly the same. Namely, they consist of the equivalence classes of $2 n$-tuples $\left(X_{1}, \ldots, X_{n}, X_{1}, \ldots, X_{n}\right)$ such that $X_{1}$ commutes with $X_{2}$. These cancel in the difference cycle $\hat{\Gamma}_{\sigma_{1}^{-2}}-\hat{\Delta}_{n}$, thus making it belong to $\hat{V}_{n}$.

One can isotope $\hat{\Gamma}_{\varepsilon \sigma}$ into $\widetilde{\Gamma}_{\varepsilon \sigma}$ using an isotopy with compact support so that $\widetilde{\Gamma}_{\varepsilon \sigma}$ is transverse to $\hat{\Gamma}_{\sigma_{1}^{-2}}-\hat{\Delta}_{n}$. The latter means precisely that $\widetilde{\Gamma}_{\varepsilon \sigma}$ stays away from $\left(S_{2} \times \Delta_{n-2}\right) / \operatorname{SO}(3)$ and is transverse to both $\hat{\Gamma}_{\sigma_{1}^{-2}}$ and $\hat{\Delta}_{n}$; a precise argument can be found in [Heusener and Kroll 1998, page 491]. We further extend this isotopy to make $\widetilde{\Gamma}_{\varepsilon \sigma}$ transverse to $\hat{V}_{n}$ so that their intersection is a naturally oriented 1-dimensional submanifold of $\hat{H}_{n}$.

The natural projection $p: V_{n} \rightarrow H_{2}$ induces a map $\hat{p}: \hat{V}_{n} \rightarrow \hat{H}_{2}$. Use a further small compactly supported isotopy of $\widetilde{\Gamma}_{\varepsilon \sigma}$, if necessary, to make $\hat{p}\left(\hat{V}_{n} \cap \widetilde{\Gamma}_{\varepsilon \sigma}\right)$ into a 1-submanifold of $\hat{H}_{2}$. The proofs of [Lin 1992, Lemmas 2.2 and 2.3] then go through with little change to give us the identity

$$
\left\langle\hat{\Gamma}_{\sigma_{1}^{-2}}-\hat{\Delta}_{n}, \hat{\Gamma}_{\varepsilon \sigma}\right\rangle_{\hat{H}_{n}}=\left\langle\hat{\Gamma}_{\sigma_{1}^{-2}}-\hat{\Delta}_{2}, \hat{p}\left(\hat{V}_{n} \cap \tilde{\Gamma}_{\varepsilon \sigma}\right)\right\rangle_{\hat{H}_{2}} .
$$

5d. Computation in the pillowcase. We first study the behavior of $\hat{p}\left(\hat{V}_{n} \cap \hat{\Gamma}_{\varepsilon \sigma}\right)$ near the corners of $\hat{H}_{2}$.

Proposition 5.3. There is a neighborhood around $A^{\prime}$ in the pillowcase $\hat{H}_{2}$ inside which $\hat{p}\left(\hat{V}_{n} \cap \hat{\Gamma}_{\varepsilon \sigma}\right)$ is a curve approaching $A^{\prime}$.

Proof. Let us consider the submanifold

$$
\Delta_{n}^{\prime}=\left\{\left(X_{1}, X_{2}, X_{3}, \ldots, X_{n} ; Y_{1}, Y_{2}, X_{3}, \ldots, X_{n}\right)\right\} \subset Q_{n} \times Q_{n}
$$

and observe that $V_{n} \cap \Gamma_{\varepsilon \sigma}=\Delta_{n}^{\prime} \cap \Gamma_{\varepsilon \sigma}$. We will show that the intersection of $\Delta_{n}^{\prime}$ with $\Gamma_{\varepsilon \sigma}$ is transversal at $(\boldsymbol{i}, \varepsilon \boldsymbol{i})=(i, \ldots, i ;-i,-i, i, \ldots, i)$. This will imply that $\Delta_{n}^{\prime} \cap \Gamma_{\varepsilon \sigma}$ is a submanifold of dimension four in a neighborhood of $(\boldsymbol{i}, \varepsilon \boldsymbol{i})$ and, after factoring out the $\mathrm{SO}(3)$ symmetry, that $\hat{p}\left(\hat{V}_{n} \cap \hat{\Gamma}_{\varepsilon \sigma}\right)$ is a curve approaching $A^{\prime}=p(\boldsymbol{i}, \varepsilon \boldsymbol{i})$.

Since $\operatorname{dim} \Delta_{n}^{\prime}=2 n+4$, the dimension of $T_{(i, \varepsilon i)}\left(\Delta_{n}^{\prime} \cap \Gamma_{\varepsilon \sigma}\right)=T_{(i, \varepsilon i)} \Delta_{n}^{\prime} \cap T_{(i, \varepsilon i)} \Gamma_{\varepsilon \sigma}$ is at least four. Therefore, checking the transversality amounts to showing that this dimension is exactly four. Write

$$
\begin{aligned}
T_{(\boldsymbol{i}, \varepsilon \boldsymbol{i})}\left(\Delta_{n}^{\prime}\right) & =\left\{\left(u_{1}, \ldots u_{n} ; v_{1}, v_{2}, u_{3}, \ldots, u_{n}\right)\right\} \subset T_{(\boldsymbol{i}, \varepsilon \boldsymbol{i})}\left(Q_{n} \times Q_{n}\right), \\
T_{(\boldsymbol{i}, \varepsilon \boldsymbol{i})}\left(\Gamma_{\varepsilon \sigma}\right) & =\left\{\left(u_{1}, \ldots, u_{n} ; d_{i}(\varepsilon \sigma)\left(u_{1}, \ldots, u_{n}\right)\right\} \subset T_{(\boldsymbol{i}, \varepsilon \boldsymbol{i})}\left(Q_{n} \times Q_{n}\right) .\right.
\end{aligned}
$$


Then $T_{(i, \varepsilon i)}\left(\Delta_{n}^{\prime}\right) \cap T_{(i, \varepsilon i)}\left(\Gamma_{\varepsilon \sigma}\right)$ consists of the vectors $\left(u_{1}, \ldots, u_{n}\right) \in T_{i} Q_{n}=T_{i} S^{2} \oplus$ $\cdots \oplus T_{i} S^{2}$ that solve the matrix equation

$$
\left[d_{i}(\sigma)\right]\left[\begin{array}{c}
u_{1} \\
u_{2} \\
u_{3} \\
\vdots \\
u_{n}
\end{array}\right]=\left[\begin{array}{c}
* \\
* \\
u_{3} \\
\vdots \\
u_{n}
\end{array}\right]
$$

since $\varepsilon=(-1,-1,1, \ldots, 1)$, we can safely replace $\left[d_{i}(\varepsilon \sigma)\right]$ by $\left[d_{i}(\sigma)\right]$. It is shown in [Long 1989] that $\left[d_{i}(\sigma)\right]$ is the Burau matrix of $\sigma$ with parameter equal to -1 . It is a real matrix acting on $T_{i} Q_{n}=\mathbb{C}^{n}$; hence all we need to show is that the space of $\left(u_{1}, \ldots, u_{n}\right) \in \mathbb{R}^{n}$ solving (11) has real dimension two. Let us write

$$
\left[d_{i}(\sigma)\right]=\left[\begin{array}{ll}
A & B \\
C & D
\end{array}\right]
$$

where $A$ is a $2 \times 2$ matrix and $D$ is an $(n-2) \times(n-2)$ matrix. Equation (11) is equivalent to

$$
[C]\left[\begin{array}{l}
u_{1} \\
u_{2}
\end{array}\right]=[1-D]\left[\begin{array}{c}
u_{3} \\
\vdots \\
u_{n}
\end{array}\right],
$$

so the proposition will follow as soon as we show that $1-D$ is invertible. The invertibility of $1-D$ is a consequence of the following two lemmas.

Lemma 5.4. Let $\sigma \in \mathscr{B}_{n}$. Then the Burau matrix of $\sigma$ with parameter -1 and the permutation matrix of $\bar{\sigma}^{-1}$ are the same modulo 2 .

Proof. According to [Birman 1974], the Burau matrix of $\sigma$ with parameter $t$ is the matrix

$$
\left.\frac{\partial \sigma\left(x_{i}\right)}{\partial x_{j}}\right|_{x_{i}=t}
$$

where the $x_{i}$ are generators of the free group and $\partial$ is the derivative in the Fox free differential calculus; see [Fox 1962]. Applying the Fox calculus we obtain

$$
\begin{aligned}
\frac{\partial \sigma\left(x_{i}\right)}{\partial x_{j}}=\frac{\partial\left(w x_{\bar{\sigma}^{-1}(i)} w^{-1}\right)}{\partial x_{j}} & =\frac{\partial w}{\partial x_{j}}+w\left(\frac{\partial\left(x_{\bar{\sigma}^{-1}(i)} w^{-1}\right)}{\partial x_{j}}\right) \\
& =\frac{\partial w}{\partial x_{j}}+w\left(\frac{\partial x_{\bar{\sigma}^{-1}(i)}}{\partial x_{j}}+x_{\bar{\sigma}^{-1}(i)} \frac{\partial w^{-1}}{\partial x_{j}}\right) \\
& =\frac{\partial w}{\partial x_{j}}+w \frac{\partial x_{\bar{\sigma}^{-1}(i)}}{\partial x_{j}}-w x_{\bar{\sigma}^{-1}(i)} w^{-1} \frac{\partial w}{\partial x_{j}},
\end{aligned}
$$


where $w$ is a word in the $x_{i}$. After evaluating at $t=-1$ and reducing modulo 2, the above becomes simply $\partial x_{\bar{\sigma}^{-1}(i)} / \partial x_{j}$, which is the permutation matrix of $\bar{\sigma}^{-1}$.

Lemma 5.5. Let $\sigma \in \mathscr{B}_{n}$ be such that $\hat{\sigma}$ is a two component link. Then $1-D$ is invertible.

Proof. Our assumption in this section has been that $\bar{\sigma}=(1, \ldots)(2, \ldots)$. We may further assume that

$$
\bar{\sigma}=(1,3,4, \ldots, k)(2, k+1, k+2, \ldots, n)
$$

by applying a sequence of first Markov moves fixing the first two strands of $\sigma$. The matrix $D(\bmod 2)$ is obtained by crossing out the first two rows and first two columns in the permutation matrix of $\bar{\sigma}$; see Lemma 5.4. This description implies that $D(\bmod 2)$ is upper diagonal, and hence so is $(1-D)(\bmod 2)$. The diagonal elements of the latter matrix are all equal to one; therefore, $\operatorname{det}(1-D)=1(\bmod 2)$ so $1-D$ is invertible.

Remark 5.6. The orientation of the component of $\hat{p}\left(\hat{V}_{n} \cap \hat{\Gamma}_{\varepsilon \sigma}\right)$ limiting to $A^{\prime}$ can be read off its description near $A^{\prime}$ given in the proof of Proposition 5.3. In particular, this orientation is independent of the choice of $\sigma$.

Proposition 5.7. There are neighborhoods around $A$ and $B^{\prime}$ in the pillowcase $\hat{H}_{2}$ that are disjoint from $\hat{p}\left(\hat{V}_{n} \cap \hat{\Gamma}_{\varepsilon \sigma}\right)$.

Proof. The arguments for $A$ and $B^{\prime}$ are essentially the same so we will only give the proof for $A$. Assuming the contrary we have a curve in $\hat{V}_{n} \cap \hat{\Gamma}_{\varepsilon \sigma}$ limiting to a reducible representation in $V_{n} \cap \Gamma_{\varepsilon \sigma}$. After conjugating if necessary, this representation must have the form

$$
\left(i, i, e^{i \varphi_{3}}, \ldots, e^{i \varphi_{n}}, i, i, e^{i \varphi_{3}}, \ldots, e^{i \varphi_{n}}\right) .
$$

Using that $\varepsilon=(-1,-1,1, \ldots, 1)$ and arguing as in the proof of Proposition 3.5, we arrive at the contradiction $i=-i$.

Proof of Theorem 2. According to Proposition 5.3, the 1-submanifold $\hat{p}\left(\hat{V}_{n} \cap \widetilde{\Gamma}_{\varepsilon \sigma}\right)$, near $A^{\prime}$, is a curve approaching $A^{\prime}$. According to Proposition 5.7, the other end of this curve must approach $B$. Therefore

$$
h\left(\sigma_{1}^{2} \sigma\right)-h(\sigma)=\left\langle\hat{\Gamma}_{\sigma_{1}^{-2}}-\hat{\Delta}_{2}, \hat{p}\left(\hat{V}_{n} \cap \widetilde{\Gamma}_{\varepsilon \sigma}\right)\right\rangle_{\hat{H}_{2}}
$$

is the same as the intersection number of an arc going from $A^{\prime}$ to $B$ with the difference cycle $\hat{\Gamma}_{\sigma_{1}^{-2}}-\hat{\Delta}_{2}$. This number is either 1 or -1 but is the same for all $\sigma$; see Remark 5.6. This is sufficient to prove that $h(\sigma)$ is the linking number up to an overall sign. 


\section{References}

[Alexander 1923] J. Alexander, "A lemma on systems of knotted curves”, Proc. Nath. Acad. Sci. 9 (1923), 93-95. JFM 49.0408.03

[Birman 1974] J. S. Birman, Braids, links, and mapping class groups, Annals of Mathematics Studies 82, Princeton University Press, 1974. MR 51 \#11477 Zbl 0305.57013

[Collin and Steer 1999] O. Collin and B. Steer, "Instanton Floer homology for knots via 3-orbifolds", J. Differential Geom. 51:1 (1999), 149-202. MR 2000j:57069 Zbl 1025.57034

[Floer 1988] A. Floer, "An instanton-invariant for 3-manifolds", Comm. Math. Phys. 118:2 (1988), 215-240. MR 89k:57028 Zbl 0684.53027

[Fox 1962] R. H. Fox, "A quick trip through knot theory", pp. 120-167 in Topology of 3-manifolds and related topics (Athens, GA, 1961), edited by M. K. Fort, Jr., Prentice-Hall, Englewood Cliffs, NJ, 1962. MR 25 \#3522

[Herald 1997] C. M. Herald, "Flat connections, the Alexander invariant, and Casson's invariant", Comm. Anal. Geom. 5:1 (1997), 93-120. MR 98i:57062 Zbl 0893.57003

[Heusener and Kroll 1998] M. Heusener and J. Kroll, "Deforming abelian SU(2)-representations of knot groups", Comment. Math. Helv. 73:3 (1998), 480-498. MR 99g:57008 Zbl 0910.57004

[Kronheimer and Mrowka 2008] P. Kronheimer and T. Mrowka, "Knot homology groups from instantons", preprint, version 2, 2008. arXiv 0806.1053v2

[Lin 1992] X.-S. Lin, “A knot invariant via representation spaces”, J. Differential Geom. 35:2 (1992), 337-357. MR 93e:57012 Zbl 0774.57007

[Long 1989] D. D. Long, "On the linear representation of braid groups", Trans. Amer. Math. Soc. 311:2 (1989), 535-560. MR 89h:20054 Zbl 0714.20019

[Ruberman and Saveliev 2004] D. Ruberman and N. Saveliev, "Rohlin's invariant and gauge theory, I: Homology 3-tori”, Comment. Math. Helv. 79:3 (2004), 618-646. MR 2006e:57020

[Taubes 1990] C. H. Taubes, "Casson's invariant and gauge theory", J. Differential Geom. 31:2 (1990), 547-599. MR 91m:57025 Zbl 0702.53017

Received July 20, 2009.

ERIC HARPER

DEPARTMENT OF MATHEMATICS

UNIVERSITY OF MIAMI

PO BOX 249085

Coral Gables, FL 33124

UNited StATES

harper@math.miami.edu

Nikolai SaVElieV

DEPARTMENT OF MATHEMATICS

UNIVERSITY OF MIAMI

PO BOX 249085

Coral Gables, FL 33124

UNITED STATES

saveliev@math.miami.edu 


\title{
PACIFIC JOURNAL OF MATHEMATICS
}

\author{
http://www.pjmath.org \\ Founded in 1951 by \\ E. F. Beckenbach (1906-1982) and F. Wolf (1904-1989)
}

\section{EDITORS}

V. S. Varadarajan (Managing Editor)

Department of Mathematics

University of California

Los Angeles, CA 90095-1555

pacific@math.ucla.edu

Vyjayanthi Chari

Department of Mathematics

University of California

Riverside, CA 92521-0135

chari@math.ucr.edu

Robert Finn

Department of Mathematics Stanford University

Stanford, CA 94305-2125

finn@math.stanford.edu

Kefeng Liu

Department of Mathematics

University of California

Los Angeles, CA 90095-1555

liu@math.ucla.edu
Darren Long

Department of Mathematics

University of California

Santa Barbara, CA 93106-3080

long@math.ucsb.edu

Jiang-Hua Lu

Department of Mathematics

The University of Hong Kong

Pokfulam Rd., Hong Kong jhlu@maths.hku.hk

Alexander Merkurjev

Department of Mathematics

University of California

Los Angeles, CA 90095-1555

merkurev@math.ucla.edu
Sorin Popa

Department of Mathematics University of California

Los Angeles, CA 90095-1555 popa@math.ucla.edu

Jie Qing

Department of Mathematics

University of California

Santa Cruz, CA 95064

qing@cats.ucsc.edu

Jonathan Rogawski

Department of Mathematics

University of California

Los Angeles, CA 90095-1555

jonr@math.ucla.edu

\section{PRODUCTION}

pacific@math.berkeley.edu

\begin{abstract}
Silvio Levy, Scientific Editor Matthew Cargo, Senior Production Editor
\end{abstract}
ACADEMIA SINICA, TAIPEI

CALIFORNIA INST. OF TECHNOLOGY

INST. DE MATEMÁTICA PURA E APLICADA

KEIO UNIVERSITY

MATH. SCIENCES RESEARCH INSTITUTE

NEW MEXICO STATE UNIV.

OREGON STATE UNIV.

\section{SUPPORTING INSTITUTIONS}

STANFORD UNIVERSITY
UNIV. OF BRITISH COLUMBIA
UNIV. OF CALIFORNIA, BERKELEY
UNIV. OF CALIFORNIA, DAVIS
UNIV. OF CALIFORNIA, LOS ANGELES
UNIV. OF CALIFORNIA, RIVERSIDE
UNIV. OF CALIFORNIA, SAN DIEGO
UNIV. OF CALIF., SANTA BARBARA

UNIV. OF CALIF., SANTA CRUZ

UNIV. OF MONTANA

UNIV. OF OREGON

UNIV. OF SOUTHERN CALIFORNIA

UNIV. OF UTAH

UNIV. OF WASHINGTON

WASHINGTON STATE UNIVERSITY

These supporting institutions contribute to the cost of publication of this Journal, but they are not owners or publishers and have no responsibility for its contents or policies.

See inside back cover or www.pjmath.org for submission instructions.

The subscription price for 2010 is US \$420/year for the electronic version, and \$485/year for print and electronic.

Subscriptions, requests for back issues from the last three years and changes of subscribers address should be sent to Pacific Journal of Mathematics, P.O. Box 4163, Berkeley, CA 94704-0163, U.S.A. Prior back issues are obtainable from Periodicals Service Company, 11 Main Street, Germantown, NY 12526-5635. The Pacific Journal of Mathematics is indexed by Mathematical Reviews, Zentralblatt MATH, PASCAL CNRS Index, Referativnyi Zhurnal, Current Mathematical Publications and the Science Citation Index.

The Pacific Journal of Mathematics (ISSN 0030-8730) at the University of California, c/o Department of Mathematics, 969 Evans Hall, Berkeley, CA 94720-3840, is published monthly except July and August. Periodical rate postage paid at Berkeley, CA 94704, and additional mailing offices. POSTMASTER: send address changes to Pacific Journal of Mathematics, P.O. Box 4163, Berkeley, CA 94704-0163.

PJM peer review and production are managed by EditFLOW ${ }^{\mathrm{TM}}$ from Mathematical Sciences Publishers.

PUBLISHED BY PACIFIC JOURNAL OF MATHEMATICS

at the University of California, Berkeley 94720-3840

A NON-PROFIT CORPORATION

Typeset in LATEX

Copyright $(\mathrm{C} 2010$ by Pacific Journal of Mathematics 


\section{PACIFIC JOURNAL OF MATHEMATICS}

Volume $248 \quad$ No. $1 \quad$ November 2010

An existence theorem of conformal scalar-flat metrics on manifolds with boundary 1

SÉRgio de Moura Almaraz

Parasurface groups

KHALID BOU-RABEE

Expressions for Catalan Kronecker products

Andrew A. H. Brown, Stephanie Van Willigenburg and Mike

ZABROCKI

Metric properties of higher-dimensional Thompson's groups

José BURILLO and SEAN CLEARY

Solitary waves for the Hartree equation with a slowly varying potential

KIRIL DATCHEV and IVAN VENTURA

Uniquely presented finitely generated commutative monoids

PEDRO A. GARCíA-SÁNCHEZ and IgnaCio OJEDA

The unitary dual of $p$-adic $\widetilde{\operatorname{Sp}(2)}$

MARCELA HANZER and IVAN MATIĆ

A Casson-Lin type invariant for links

ERIC HARPER and NIKOLAI SAVELIEV

Semiquandles and flat virtual knots

ALLISON HENRICH and SAM NELSON

Infinitesimal rigidity of polyhedra with vertices in convex position

IVAN IZMESTIEV and JEAN-MARC SCHLENKER

Robust four-manifolds and robust embeddings

VYACHESLAV S. KRUSHKAL

On sections of genus two Lefschetz fibrations

SINEM ÇELIK ONARAN

Biharmonic hypersurfaces in Riemannian manifolds

YE-LIN OU

Singular fibers and 4-dimensional cobordism group 